\title{
Role and Responsibility of Nurses in Central Line - Insertion and Maintenance
}

\author{
R. Surendra Naik ${ }^{1}$, Avadhesh Kumar $\operatorname{Yadav}^{2}$, Rajendra Kumar Sahu \\ Ram Niwas Sharma ${ }^{4}$
}

\author{
${ }^{1}$ Nursing Officer-A (MHM) (Officer In-Charge Surgical ICU), Mahamana Pandit Madan Mohan Malviya \\ Cancer Centre Varanasi Uttar Pradesh 221005. \\ ${ }^{2}$ Nursing Officer-B, Officer In Charge (Pediatric and Laser Surgery), Mahamana Pandit Madan Mohan Malviya \\ Cancer Centre Varanasi Uttar Pradesh 221005, (A Unit of Tata Memorial Centre Mumbai, Department of \\ Atomic Energy) \\ ${ }^{3}$ Nursing Officer-A, M.Sc. (MHN), Mahamana Pandit Madan Mohan Malviya Cancer Centre Varanasi \\ Uttar Pradesh 221005. \\ ${ }^{4}$ Nursing Officer-B, Officer In Charge, Mahamana Pandit Madan Mohan Malviya Cancer Centre Varanasi \\ Uttar Pradesh 221005, (A Unit of Tata Memorial Centre Mumbai, Department of Atomic Energy)
}

Corresponding Author: Rajendra Kumar Sahu

\begin{abstract}
Introduction -A central venous catheter (CVC) is thin, a flexible tube (catheter) that is placed into a large vein above the Heart. It may be inserted through A vein in the Neck, (internal jugular) chest (subclavian vein. Axillary vein) groin (femoral vein), or through veins in the arms known as a PICC, or peripherally inserted central catheters.
\end{abstract}

Site- Internal jugular vein, subclavian vein, axillary vein, femoral veins, the best approach or access point for Central line insertion.

Indications - The indications for central venous access are broad and are often situational. Inability to obtain venous access in emergent situations, chemotherapy administration, medications administration (Vasopressors. inotropic administration Total Parental nutrition administration, Hemodynamic monitoring are common indications for CVC insertion.

Contraindication- Local cellulitis, Low platelet count, Local infections, Thrombocytopenia, Congenital anomalies, Trauma are common contraindications of CVC insertion.

Complications - Numerous potential complications can occur during the procedural placement of a central venous catheter, but also as a result of the indwelling equipment. Arrhythmias, Arterial puncture, Pulmonary puncture with or without resultant pneumothorax, Bleeding - hematoma formation, which can obstruct the airway, Tracheal injury, Air emboli during venous puncture or removal of the catheter, Pulmonary embolism, Local cellulitis, Catheter infection, Cardiac tamponade, Intravascular loss of guidewire, Hamo thorax, Phrenic nerve injury, Brachial plexus injury, Cerebral infarct from carotid artery cannulation, Bladder perforation, Bowel perforation, Sterile Thrombophlebitis.

Post-procedural complications: Catheterrelated bloodstream infections - bacterial or fungal, Central vein stenosis, Thrombosis, Delayed bleeding with multiple attempts in a coagulopathic patient Clinical

Significance - Ensure that sterile products are not contaminated and that there is no evidence of damage to the packaging. Follow sterile procedures at all times. Central line infections can be a serious and life-threatening illness. Always ensure that the catheter is appropriately placed through one or several methods: radiographic evidence, measurement of CVP, or by analyzing a venous blood gas. Never use excessive force during any part of this procedure. It will lead to damage to local structures.

Nursing Responsibility - After a CVC placement, nurses are responsible for maintaining, monitoring, and utilizing central venous catheters. The assigned nurse must check complications such as infections, hematoma, thrombosis of the catheter, and signs 
of pneumothorax and bleeding. Nurses are also responsible for ensuring that the site is maintained in a clean and sterile fashion. Daily inspection of the access site and device patency should be performed during nursing rounds. In particular, nursing officers must disinfect injection ports, catheter hubs, and needleless connectors with institutionally approved antiseptics. Intravenous administration sets should be changed regularly per hospital policy. The site should be checked for bleeding, hematoma formation, and signs of cellulitis, which include erythema, purulent drainage, and/or warmth. Dressings should be changed if visibly soiled. This must be performed with proper sterile technique.

Keywords - CVC, Central Line, Central venous catheter

\section{INTRODUCTION}

A central venous catheter $(\mathrm{CVC})$ is thin, a flexible tube (catheter) that is placed into a large vein above the Heart. It may be inserted through a vein in the Neck, (internal jugular) chest (subclavian vein. Axillary vein) groin (femoral vein), or through veins in the arms known as a PICC, or peripherally inserted central catheters It's also called central venous line or central line some catheters have 2 are 3 tubes (double are triple lumen catheters.

A central venous catheter is an invasive catheter that is peripherally inserted into the (veins large and small veins) mostly preferable in the internal jugular and subclavian or femoral veins, the term central line first described in 1929 after that venous access following rapidly increased as like an important device that mainly used in cardiac surgeries for central venous pressure monitoring and pulmonary artery pressure monitoring, Glenn pressure monitoring purposes useful central line mostly for hemodynamic monitoring for critically ill patients in critical care units Renal Replacement purpose for emergency resuscitation using in critical care units.

Anatomy and physiology: Central venous line mainly following three sites
Internal jugular vein, subclavian vein, axillary vein, femoral veins, the best approach or access point varies always depend up on patient condition and clinical needs IJV or EJV this vein lie both sides of neck this vein carry deoxygenated blood from head, face, and neck towards the heart both veins unite to subclavian vein, the Internal Jugular approach allows for ultrasound Guidance approach While following this approach insertion related complication reduced disadvantages includes in this approach is arterial puncture, infection rates is higher than subclavian approach the internal jugular site more preferable for temporary dialysis purpose and this site not a first choice in post tracheostomies or post vascular surgical patients adjacent anatomy includes carotid artery, phrenic and vagus nerves pulmonary apex, thoracic duct The External Jugular veins are located outside of the SCM, This veins are easily visible from the surface making it easy to locate, uses of External Jugular Veins is considered advanced skill the veins close to proximal carotid artery, the adjacent anatomy includes carotid artery, phrenic artery, pulmonary apex thoracic duct the Sub clavian veins are lies below the clavicle which is located above the $1^{\text {st }}$ Rib An extension of the large axillary vein, it begins at outer border of the first Rib passage over the Rib extend to inner border of clavicle, for subclavian vein adjacent anatomy includes Right Lymphatic duct, costoclavicular ligament, first Rib Axillary veins is a continuation of brachial veins cephalic veins continuous to becomes subclavian veins at Lateral border of first Rib, Adjacent anatomy includes Cephalic vein, subclavian vein, First Rib ,clavicle femoral vein is the main vein in the leg and accompanies to the femoral artery in the femoral sheath, it stretches from the adductor canal to the internal jugular ligament at which point it becomes the external iliac veins the femoral nerve which less outside the femoral sheath 


\section{Indications}

The indications for central venous access are broad and are often situational (1). In no particular order, they include:

- Inability to obtain venous access in emergent situations.

- For critically ill patients (ICU patients with septic shock. Cardiac surgery patients mostly required in pediatric patients)

- For chemotherapy administration medications administration (Vasopressors. inotropic administration Total Parenteral nutrition administration

- Hemodynamic monitoring, including central venous pressures. Glenn pressures

- For venous interventions, including inferior vena cava filter placement, and cardiac surgery patients may need

- For a long time, pain management

- For hypertonic fluid administration

- For Emergency Renal Replacement Therapy
- Resuscitation of intravascularly depleted patients

\section{Contraindication}

- Local cellulitis

- Low platelet count

- Local infections

- Avoid intracranial pressure raised when femoral approach required

- Patient Non-compliance

- Thrombocytopenia

- Congenital anomalies

- Trauma

Typically, patients who have an international normalized ratio (INR) of greater than 3.0 patients who have low platelets count less than 150000 this patient may have an increases Risk of bleeding It may be warned sign to transfuse platelets or fresh frozen plasma the central line is contraindicated in coagulopathy's patients,

\section{Sizes of Central line:}

\begin{tabular}{|c|c|c|c|c|c|c|}
\hline & & \multicolumn{2}{|c|}{ INDWELLING CATHETER } & \multicolumn{3}{|c|}{ GUIDE WIRE } \\
\hline \multicolumn{2}{|c|}{ Description } & $\begin{array}{c}\text { Specification } \\
\text { (Size) }\end{array}$ & $\begin{array}{c}\text { Length } \\
(\mathrm{mm})\end{array}$ & $\begin{array}{c}\text { Diameter } \\
\text { (inch } / \mathrm{mm} \text { ) }\end{array}$ & $\begin{array}{l}\text { Length } \\
(\mathrm{cm} / \mathrm{mm})\end{array}$ & Needle \\
\hline \multirow{12}{*}{$\begin{array}{l}\text { Single } \\
\text { Lumen }\end{array}$} & 11041 & $3 F R / 20 G(D)$ & 130 & $0.025 / 0.64$ & $50 / 500$ & $20 \mathrm{G}$ \\
\hline & 11042 & $3 F R / 20 G(D)$ & 150 & $0.025 / 0.64$ & $50 / 500$ & $20 \mathrm{G}$ \\
\hline & 11043 & $3 F R / 20 G(D)$ & 200 & $0.025 / 0.64$ & $50 / 500$ & $20 \mathrm{G}$ \\
\hline & 11017 & 4FR / 18G (D) & 130 & $0.025 / 0.64$ & $50 / 500$ & $18 \mathrm{G}$ \\
\hline & 11018 & $4 \mathrm{FR} / 18 \mathrm{G}(\mathrm{D})$ & 150 & $0.025 / 0.64$ & $50 / 500$ & $18 \mathrm{G}$ \\
\hline & 11019 & 4FR / 18G (D) & 200 & $0.025 / 0.64$ & $50 / 500$ & $18 \mathrm{G}$ \\
\hline & 11011 & $5 F R / 16 G(D)$ & 160 & $0.035 / 0.89$ & $50 / 500$ & $18 \mathrm{G}$ \\
\hline & 11012 & $5 F R / 16 G(D)$ & 200 & $0.035 / 0.89$ & $50 / 500$ & $18 \mathrm{G}$ \\
\hline & 11013 & $5 F R / 16 G(D)$ & 300 & $0.035 / 0.89$ & $70 / 700$ & $18 \mathrm{G}$ \\
\hline & 11014 & 6FR / 14G (D) & 160 & $0.035 / 0.89$ & $50 / 500$ & $18 \mathrm{G}$ \\
\hline & 11015 & $6 \mathrm{FR} / 14 \mathrm{G}(\mathrm{D})$ & 200 & $0.035 / 0.89$ & $50 / 500$ & $18 \mathrm{G}$ \\
\hline & 11016 & 6FR / 14G (D) & 300 & $0.035 / 0.89$ & $70 / 700$ & $18 \mathrm{G}$ \\
\hline \multirow{8}{*}{$\begin{array}{l}\text { Double } \\
\text { Lumen }\end{array}$} & 11027 & $4 F R / 22(P)-20 G(D)$ & 130 & $0.018 / 0.46$ & $50 / 500$ & $21 \mathrm{G}$ \\
\hline & 11028 & $4 F R / 22(P)-20 G(D)$ & 160 & $0.018 / 0.46$ & $50 / 500$ & $21 \mathrm{G}$ \\
\hline & 11021 & $7 F R / 16 \mathrm{G}(\mathrm{P})-16 \mathrm{G}(\mathrm{D})$ & 160 & $0.035 / 0.89$ & $50 / 500$ & $18 \mathrm{G}$ \\
\hline & 11022 & $7 F R / 16 \mathrm{G}(\mathrm{P})-16 \mathrm{G}(\mathrm{D})$ & 200 & $0.035 / 0.89$ & $50 / 500$ & $18 \mathrm{G}$ \\
\hline & 11023 & $7 F R / 16 G(P)-16 G(D)$ & 300 & $0.035 / 0.89$ & $70 / 700$ & $18 \mathrm{G}$ \\
\hline & 11024 & $7 F R / 14 G(P)-18 G(D)$ & 160 & $0.025 / 0.64$ & $50 / 500$ & $18 \mathrm{G}$ \\
\hline & 11025 & $7 F R / 14 G(P)-18 G(D)$ & 200 & $0.025 / 0.64$ & $50 / 500$ & $18 \mathrm{G}$ \\
\hline & 11026 & $7 F R / 14 G(P)-18 G(D)$ & 300 & $0.025 / 0.64$ & $70 / 700$ & $18 \mathrm{G}$ \\
\hline \multirow{5}{*}{$\begin{array}{l}\text { Triple } \\
\text { Lumen }\end{array}$} & 11034 & $5.5 F R / 22 G(P)-20 G(D)-22 G(M)$ & 80 & $0.018 / 0.46$ & $50 / 500$ & $21 \mathrm{G}$ \\
\hline & 11035 & $5.5 F R / 22 \mathrm{G}(\mathrm{P})-20 \mathrm{G}(\mathrm{D})-22 \mathrm{G}(\mathrm{M})$ & 160 & $0.018 / 0.46$ & $50 / 500$ & $21 \mathrm{G}$ \\
\hline & 11031 & 7FR / 18G(P) - 16G(D) - 18G(M) & 160 & $0.035 / 0.89$ & $50 / 500$ & $18 \mathrm{G}$ \\
\hline & 11032 & $7 F R / 18 G(P)-16 G(D)-18 G(M)$ & 200 & $0.035 / 0.89$ & $50 / 500$ & $18 \mathrm{G}$ \\
\hline & 11033 & $7 F R / 18 G(P)-16 G(D)-18 G(M)$ & 300 & $0.035 / 0.89$ & $70 / 700$ & $18 \mathrm{G}$ \\
\hline
\end{tabular}




\section{Equipment}

There are various types of manufactures central line catheters kits is available in different types of catheters basically required equipment are following that ultrasound machine with linear probe .camera cover, jelly, and personal protective equipment's (cap mask gown gloves shoo cover ) ICU pack which may include drapes (small towel big towel whole towel drape) gown pack sterile surgical gloves suture material $10 \mathrm{cc}$ syringe local(lignocaine $2 \%$ ) sterile needle $100 \mathrm{ml}$ normal saline gauze pieces and Gamze pad central line Tigard rum proper biomedical waste management.

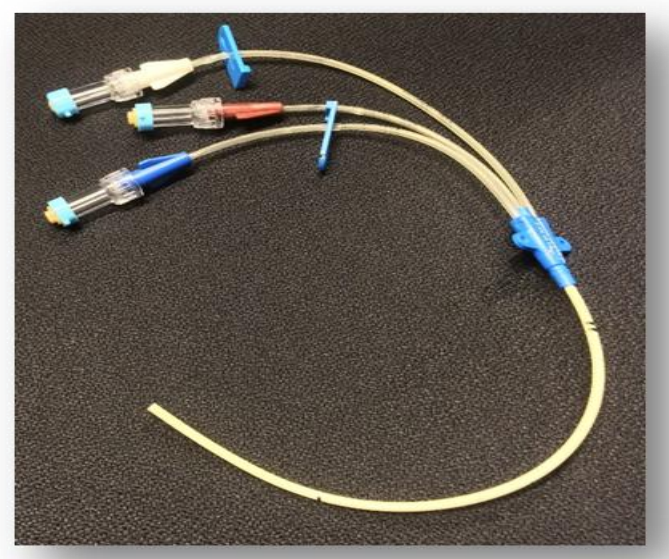

Non-sterile products:

* Cap surgeon

* Mask with face shield

* Biomedical waste bins (red yellow. Block) for safe disposal of waste management

\section{Sterile products:}

* Personal protective equipment: including gloves, gown

* ICU pack (small towel. Big towel. Whole towel drape)

* Gown pack (sterile gown with rapper)

* Surgical gloves are an appropriate size.

* Gauze $(4 \times 4)$

* Chlorohexidine, bactoprep

* Sterile ultrasound probe covers with sterile ultrasound gel

* Camera cover

* Biopath

* "Lure locks" or catheter caps for each lumen

Central venous catheter kit, which generally includes:

* Central venous catheter (triple-lumen, dual-lumen, or large bore single-lumen)

* 18-gauge introducer needle, with a syringe

* \#11 blade Scalpel

* Guidewire

* Venodilator

* Suture material (generally 3-0 silk suture with a straight needle or a needle driver)

* Normal saline for locking purpose

* $2 \%$ lidocaine, small gauge needle (25 or 27 gauge), syringe

* Ultrasound machine with a highfrequency linear transducer

* Camera cover

* Jell for probe

* Sharp container

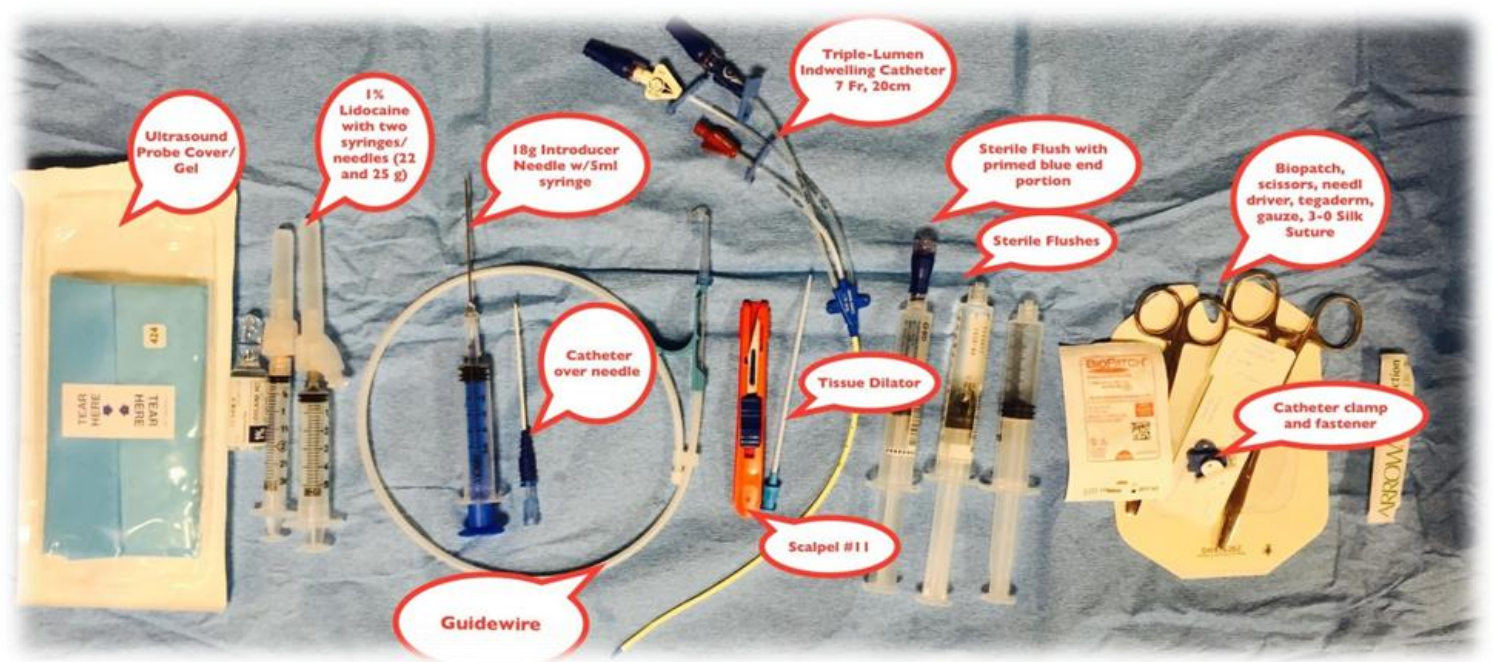




\section{ICU INTENSIVIST:}

CVC insertion should be performed by a proceduralist skilled in the technique and a nurse to assist.

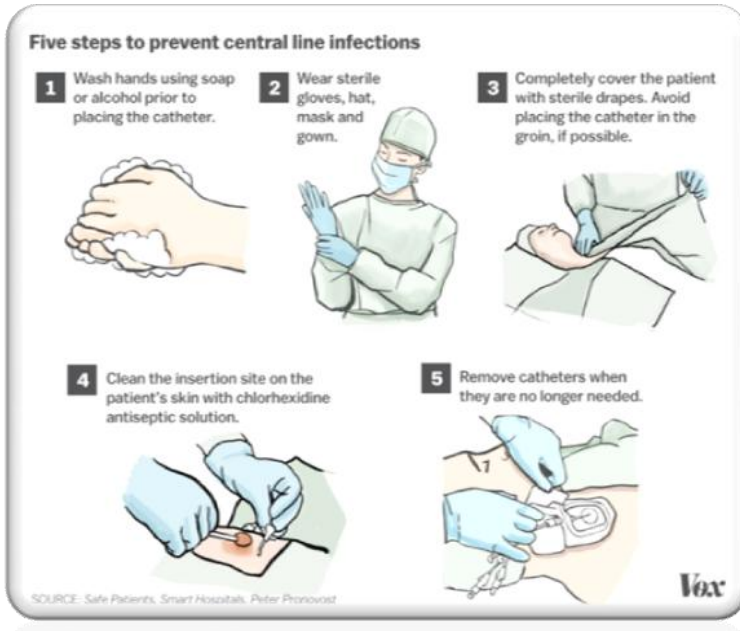

\section{Preparation}

First explain the need for central line insertion and assess The patient and family understanding.

Explain to family members about central line procedure, benefits, and complications and obtain informed consent.

Examine the patient's past medical history example past surgeries related to the chest neck are femoral region.

Determine the patient medical history related to pneumothorax or emphysema.

Assess the intended site (IJV. EJV. Subclavian. Axillary. femoral).

Perform the procedure for this need to arrange all equipment needed for insertion of CVC line.

Prepare ultrasound machines with the linear probe with jelly and arrange all personnel protective equipment (cap mask glove sterile gown face shield).

Place the waterproof pad beneath the site to be accessed and make sure that patient is in Trendelenburg position.

Prepare the skin with an antiseptic solution (2\%chlorohexidine). Perform hand hygiene and apply sterile gown and gloves.
Apply sterile drapes over the patient with the exposure of site only. Open the CVC kit in a sterile manner.

Before insertion assesses the site and checks the landmark and identify the vein and mark if Necessary.

Instruct the patient to turn his or her head slightly away from the insertion site.

Apply local 2\% Lignocaine. Place the sterile probe over the ultrasound equipment and locate the vessel.

\section{Technique}

Place the patient in the Trendelenburg position and apply bolster. Clean the area with chlorhexidine $2 \%$.

Use Seldinger technique to puncture the skin and advanced the needle while maintaining slight negative pressure with a syringe until the free flow of blood is returned. Instruct the patient to hold his breath.

Insert the guidewire into the needle then remove the needle.

Insert the dilator in the vessel with the help of a guidewire. Dilate the vessel with the help of a dilator and observe the bleeding pattern. Hold the guidewire and gently remove the dilator.

Insert the central venous catheter with the help of a guidewire. Check the backflow of each lumen and flush with normal saline all lumen. "Lure locks" may be attached to the end of each port and clean with $2 \%$ chlorhexidine solution.

Fix the central venous catheter by placing sutures, a bio-patch should be placed between the catheter hub and the skin, and a sterile occlusive dressing should be placed over the catheter/skin entry site.

Sterile drapes and soiled non-sharp products should be disposed of as per hospital policy. All sharps should be placed in sharps bins. The patient should be placed back into a position of comfort, and the Physician should verify that the line is appropriately placed within a central vein.

Confirmation of catheter in situ should be done by X-ray. 
Document procedure details with date and time in patients' records, maintain asepsis as per infection control committee guidelines. A Central line-related bloodstream infection (CLABSI) chart should be maintained.

\section{Complications}

Numerous potential complications can occur during the procedural placement of a central venous catheter, but also as a result of the indwelling equipment.

\section{Procedural complications:}

* Arrhythmias - typically ventricular or bundle branch blocks due to guidewire irritation of the atria or ventricle

* Arterial puncture

* Pulmonary puncture with or without resultant pneumothorax

* Bleeding - hematoma formation, which can obstruct the airway

* Tracheal injury

* Air emboli during venous puncture or removal of the catheter

* Pulmonary embolism

* Local cellulitis

* Catheter infection

* Cardiac tamponade

* Intravascular loss of guide wire

* Hemothorax

* Phrenic nerve injury

* Brachial plexus injury

* Cerebral infarct from carotid artery cannulation

* Bladder perforation

* Bowel perforation

* Sterile Thrombophlebitis

\section{Post-procedural complications:}

* Catheter-related bloodstream infections - bacterial or fungal

* Central vein stenosis

* Thrombosis

* Delayed bleeding with multiple attempts in a coagulopathic patient

\section{Clinical Significance}

When performed properly, the insertion of a central venous catheter is safe, efficacious, and potentially life-saving. However, certain clinical pearls should be at the forefront of the proceduralist's mind when performing this procedure.

* Whenever possible, take the time to fully prepare for the procedure, and assure that all necessary personnel and equipment are in the room and readily available. Lack of preparation will compound any potential complications that may be encountered.

* Ensure that sterile products are not contaminated and that there is no evidence of damage to the packaging. Follow sterile procedures at all times. Central line infections can be a serious and life-threatening illness.

* When using the IJ or SC site for access, be sure to obtain a stat portable chest $\mathrm{x}$-ray immediately after line placement to ensure there is no pneumothorax and that the line terminates in the superior vena cava.

* If one has a failed attempt at the IJ site and needs to seek access at another site, the ipsilateral subclavian is preferred, given the risk of bilateral pneumothoraxes with an attempt at the contralateral internal jugular vein. One may anticipate this possibility by cleaning and prepping both the IJ and SC site on the side of the procedure.

* If unsure of the placement of a guidewire within the vein, and limited views on ultrasound, manometry is a useful tool to establish that the guidewire is within the venous system. However, in shock states, where arterial pressure is low, this may be falsely reassuring.

* Subclavian access does appear to have fewer infections but potentially higher procedural complications, especially if performed by a clinician with limited experience.

* The internal jugular, subclavian, and femoral veins have higher success rates and fewer complications when access is performed with ultrasound. 
The clinician must maintain hold of the guidewire at all times while it is inside the patient. The wire can be lost inside the patient and may migrate into the right ventricle or inferior vena cava, leading to additional invasive procedures to recover the wire.

* Always ensure that the catheter is appropriately placed through one or several methods: radiographic evidence, measurement of CVP, or by analyzing a venous blood gas.

* Never use excessive force during any part of this procedure. It will lead to damage to local structures.

\section{Nursing Responsibility \\ After procedure}

* After a CVC placement, nurses are responsible for maintaining, monitoring, and utilizing central venous catheters.

* The assigned nurse must check complications such as infections, hematoma, thrombosis of the catheter, and signs of pneumothorax and bleeding.

* Nurses are also responsible for ensuring that the site is maintained in a clean and sterile fashion.

* Beyond the immediate complications of the procedure itself, the nursing officer must be immediately aware of any ongoing issues and delayed complications.

* Their role in the interprofessional team is of monumental importance in maintaining the central venous catheter and recognizing potential complications.

* Clear communication between all team members is essential to appropriate patient care.

* The clinician should inform the nurse as soon as the proper placement of the CVC is confirmed, and nursing should wait for this confirmation before using the line to administer medications. Both the nurse and the clinician should be aware of and keep track of when the line was placed. CVC's are temporary, and complication rates increase when lines are left in too long.

\section{Daily care of Patient with Central Line}

* Daily inspection of the access site and device patency should be performed during nursing rounds.

* In particular, nursing officers must disinfect injection ports, catheter hubs, and needleless connectors with institutionally approved antiseptics.

* Intravenous administration sets should be changed regularly per hospital policy.

* The site should be checked for bleeding, hematoma formation, and signs of cellulitis, which include erythema, purulent drainage, and/or warmth.

* Dressings should be changed if visibly soiled. This must be performed with proper sterile technique.

* Importantly, any manipulation of the catheter site should be done using a sterile procedure. A bouffant cap, mask, and sterile gloves must be worn to minimize infection.

* The site should be cleaned with approved antiseptics, allowed to dry, and a sterile occlusive dressing must be replaced.

* At interprofessional team rounds, there should be a daily discussion about whether or not the central venous catheter is still indicated. If deemed unnecessary for further management, the central venous catheter should be removed expeditiously.

\section{Safety Guidelines while central line care}

1. Wash hands before doing any central line care and wear gloves.

2. Always keep a clean and dry dressing over the central line.

3. Follow the instructions for cleaning the cap and using the sterile equipment.

4. Always keep sharp objects away from the central line.

5. If the central line is hard to flush do not try to flush inform the intensivist. 
6. Maintain CLABSIS bundles record date of insertion and day of the catheter.

7. As per CDC guidelines use only sterile to access catheters.

8. Immediately replace dressings that are wet, solid. Or dislodged

9. Perform routine dressing changes using aseptic technique with clean or sterile gloves

10. Change gauze dressing at least every two days or semipermeable dressing at least every seven days

11. For patients 18 years of age or older, use chlorhexidine impregnated dressing with FDA cleared label that specific a clinical indication for reducing CLABSIS for short-term non-tunneled catheters unless the facility demonstrating success at preventing CLABSIS with baseline prevention practices.

12. Change administration sets for continuous infusions no more frequently than every 24 hours.

13. If blood products or fat emulsions are administered change tubing every 24 hours

14. Perform a daily audit to assess whether each central line is still needed are not

15. Provide a checklist to the clinician to ensure adherence to aseptic insertion practices.

16. Use hospital-specific or collaborativebased performance measures to ensure compliance with recommended practices.

17. Educate health care workers about indications for a central line, proper procedures for insertion and maintenance, and appropriate infection prevention measures.

Acknowledgement: None

Conflict of Interest: None

Source of Funding: None

\section{REFERENCES}

1. Beheshti MV. A concise history of central venous access. Tech Vasc Interv Radiol. 2011 Dec;14(4):184-5. [PubMed]

2. BOLT W, KNIPPING HW. [Congratulations to Werner Forssmann on winning the 1956 Nobel prize for medicine]. Med Klin. 1956 Dec 07;51(49): 2073-6. [PubMed]

3. Konner K. History of vascular access for hemodialysis. Nephrol Dial Transplant. 2005 Dec;20(12):2629-35. [PubMed]

4. http"//booksite.elevevier.com/97803233766 2

5. Ipe TS, Marques MB. Vascular access for therapeutic plasma exchange. Transfusion. 2018 Feb;58 Suppl 1:580-589. [PubMed]

6. American Society of Anesthesiologists Task Force on Central Venous Access. Rupp SM, Apfelbaum JL, Blitt C, Caplan RA, Connis RT, Domino KB, Fleisher LA, Grant S, Mark JB, Morray JP, Nickinovich DG, Tung A. Practice guidelines for central venous access: a report by the American Society of Anesthesiologists Task Force on Central Venous Access. Anesthesiology. 2012 Mar;116(3):539-73. [PubMed]

7. Suess EM, Pinsky MR. Hemodynamic Monitoring for the Evaluation and Treatment of Shock: What Is the Current State of the Art? Semin Respir Crit Care Med. 2015 Dec;36(6):890-8. [PubMed]

\section{AUTHORS' PROFILE:}

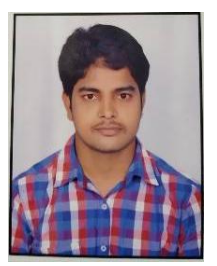

Mr. R. Surendra Naik

Nursing officer-A (MHM) (Officer In-charge Surgical ICU) Mahamana Pandit Madan Mohan Malviya Cancer Centre Varanasi Uttar Pradesh 221005.

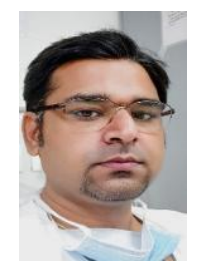

Mr. Avadhesh Kumar Yadav

Nursing officer-B, Officer in charge (Pediatric and laser Surgery)

Mahamana Pandit Madan Mohan Malviya Cancer Centre Varanasi Uttar Pradesh 221005, (A unit of Tata Memorial Centre Mumbai, Department of Atomic Energy) 


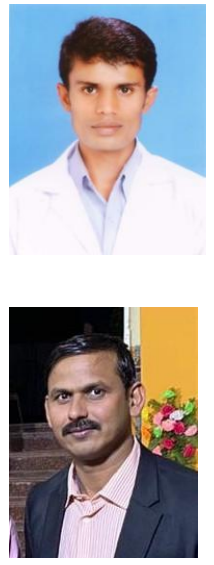

Mr. Rajendra Kumar Sahu

Nursing officer-A

M.Sc. (MHN)

Mahamana Pandit Madan Mohan

Malviya Cancer Centre Varanasi Uttar Pradesh 221005.

\section{Mr. Ram Niwas Sharma}

Nursing officer-B, Officer in charge

Mahamana Pandit Madan Mohan Malviya Cancer Centre Varanasi Uttar Pradesh 221005, (A unit of Tata Memorial Centre Mumbai, Department of Atomic Energy)
How to cite this article: R. Surendra Naik, Yadav AK, Sahu RK et.al. Role and responsibility of nurses in central line insertion and maintenance. International Journal of Research and Review. 2021; 8(9): 252-260. DOI: https://doi.org/10.52403/ijrr. 20210935 